\title{
Application of matrix-assisted laser desorption ionization time-of-flight mass spectrometry for identification of coagulase-negative staphylococci isolated from milk of cows with subclinical mastitis
}

T. Banach', M. Bochniarz'2, P. Lyp' ${ }^{1}$, L. Adaszek1, W. Wawron², B. Furmaga ${ }^{3}$, M. Skrzypczak ${ }^{4}$, J. Ziętek ${ }^{1}$, S. Winiarczyk ${ }^{1}$

${ }^{1}$ Department of Epizootiology and Infectious Diseases, Faculty of Veterinary Medicine, University of Life Sciences, Głęboka 30, 20-612 Lublin, Poland

${ }^{2}$ Department and Clinic of Animal Reproduction, Faculty of Veterinary Medicine, University of Life Sciences, Akademicka 12, 20-950 Lublin, Poland

${ }^{3}$ Sub-Department of Toxicology and Environmental Protection, Department of Preclinical Veterinary Sciences, Faculty of Veterinary Medicine, University of Life Sciences, Akademicka 12, 20-950 Lublin, Poland

${ }^{4}$ Second Department of Gynecology, Prof. F. Skubiszewski University School of Medicine, Jaczewskiego 8, 20-090 Lublin, Poland

\begin{abstract}
The aim of this study was to use matrix-assisted laser desorption ionization time-of-flight mass spectrometry (MALDI-TOF MS) for the identification of coagulase-negative staphylococci (CNS) isolated from the milk of cows with subclinical mastitis. The study material consisted of 33 isolates of CNS, identified by the results of API Staph tests, obtained from the milk of cows with subclinical mastitis. Based on the spectra analyses, MALDI-TOF MS tests of 33 bacterial samples allowed identification of the microorganisms in 27 cases $(81.8 \%)$. The most frequent cause of subclinical mastitis was found to be Staphylococcus sciuri (39\%), while S. vitulinus was detected in $15 \%$ of the milk samples. The results obtained indicate that MALDI-TOF MS can be used for the identification of CNS isolated from bovine mastitis as a method supplementary to biochemical tests.
\end{abstract}

Key words: coagulase-negative staphylococci, cows, MALDI-TOF MS, milk

Correspondence to: Ł. Adaszek, e-mail: ukaszek0@wp.pl, tel.: +48 814456192 


\section{Introduction}

Accurate bacterial identification is important in the case of outbreaks of infectious diseases, and plays a crucial role in diagnosis and effective treatment. Matrix-assisted laser desorption/ionization time-of-flight mass spectrometry (MALDI-TOF MS) is a new technology for the routine identification of bacteria in clinical microbiology laboratories (Bizzini and Greub 2010, Biswas et al. 2012). Mass spectrometry has been used for several decades in chemistry, with Anhalt and Fenselau (1975) first proposing in 1975 that bacterial characterization could be achieved using this technique. Because MALDI-TOF MS detects a large spectrum of proteins, the technique is able to discriminate between closely related species and to classify organisms at the species level (Fox 2006, Biswas et al. 2012). Much of the work using MALDI-TOF MS for microbial identification has focused on demonstrating that reproducible mass spectra can be obtained using intact cells and by developing algorithms for the interpretation and comparison of these spectra (Bright et al. 2002, Biswas et al. 2012, Tomazi et al. 2014).

MALDI-TOF MS is a quick method that has the potential to replace or complement conventional phenotypic identification of most bacterial strains isolated in clinical microbiology laboratories. Within a short period the technique has become widely adopted and has been integrated into many clinical microbiology laboratories (Carbonnelle et al. 2010, Biswas et al. 2012, Moser et al. 2013). By testing colonies, it takes only a few minutes to obtain the correct identification, making it not only possible to identify microorganisms at the species level but sometimes at the sub-species and strain levels as well, allowing detection of epidemic lineages. In addition, some antibiotic resistance might be detected as well as some bacterial toxins (Seng et al. 2010). The databases are continuously being updated, and MALDI-TOF MS represents a fast and inexpensive technology for bacterial species identification (Biswas et al. 2012).

Mastitis is the most frequent and expensive disease for the dairy cow industry. It negatively affects the economic effectiveness of farms and the quality of milk. About 150 species of microorganisms have been found as etiological agents (Malinowski et al. 2006). For many decades, coagulase-negative staphylococci, widely spread in the natural environment and colonizing the skin and mucosa of animals and humans, have been considered non-pathogenic. At present, they are a predominant etiological factor for bovine mastitis in many countries (Taponen et al. 2007, Pyörälä and Taponen 2009, Persson Waller et al. 2011, Bochniarz et al. 2013). CNS can cause clinical mastitis, although in the majority of cases they are responsible for subclinical mastitis (Taponen et al. 2007, Persson Waller et al. 2011).

The aim of this study was to use the MALDI-TOF MS method in the identification of coagulase-negative staphylococci isolated from the milk of cows with subclinical mastitis.

\section{Materials and Methods}

The study material consisted of 33 isolates of CNS obtained from the milk of cows with subclinical mastitis.

The types of mastitis were classified according to the standard principles, based on clinical examinations, milk bacteriological testing and somatic cell counts (SCC) in milk samples (using a Fossomatic device - Denmark). An SCC greater than 200,000 per milliliter of milk and the presence of bacteria in bacteriological cultures without general symptoms were considered to be subclinical mastitis (Moon et al. 2007).

The milk was bacteriologically tested according to generally accepted procedures. The milk samples were brought to room temperature, thoroughly mixed and cultured on an agar medium (BTL, Łódź, Poland) supplemented with sterile, defibrinated sheep blood (5\% of the agar solution volume). After a 24-hour incubation period at $37^{\circ} \mathrm{C}$ under aerobic conditions, any pathogens were initially identified based on colony morphology and Gram-stained microscopic specimens. The CNS were identified to the species level by the API Staph System (bioMerieux Inc., Hazelwood, MO, USA).

MALDI-TOF MS. The bacteria were prepared for mass spectrometry analysis according to a standard extraction protocol using formic acid, as recommended by Bruker (MALDI Biotyper Protocol Guide, 2015). One single bacterial colony was suspended in $1.2 \mathrm{ml}$ of $75 \%$ ethanol, centrifuged twice and then dried at room temperature. The pellet obtained was mixed with $70 \%$ formic acid ( 1 to $80 \mu \mathrm{l}$ ), and then suspended in the same volume of acetonitrile. A total of $0.5 \mu \mathrm{l}$ of the prepared material (full extraction of bacterial proteins) was applied to an MTP 384 ground steel target (Bruker Daltonics). An HCCA ( $\alpha$-Cyano-4-hydroxycinnamic acid) matrix solution (suspended in a standard solution recommended by the manufacturer, Bruker Daltonics) was placed on each dosed bacterial sample. The recommended Bruker Bacterial Test Standard (BTS) was applied in a configuration such that one location of the standard was positioned at the center of 4 bacterial sample locations. Calibration was performed using 


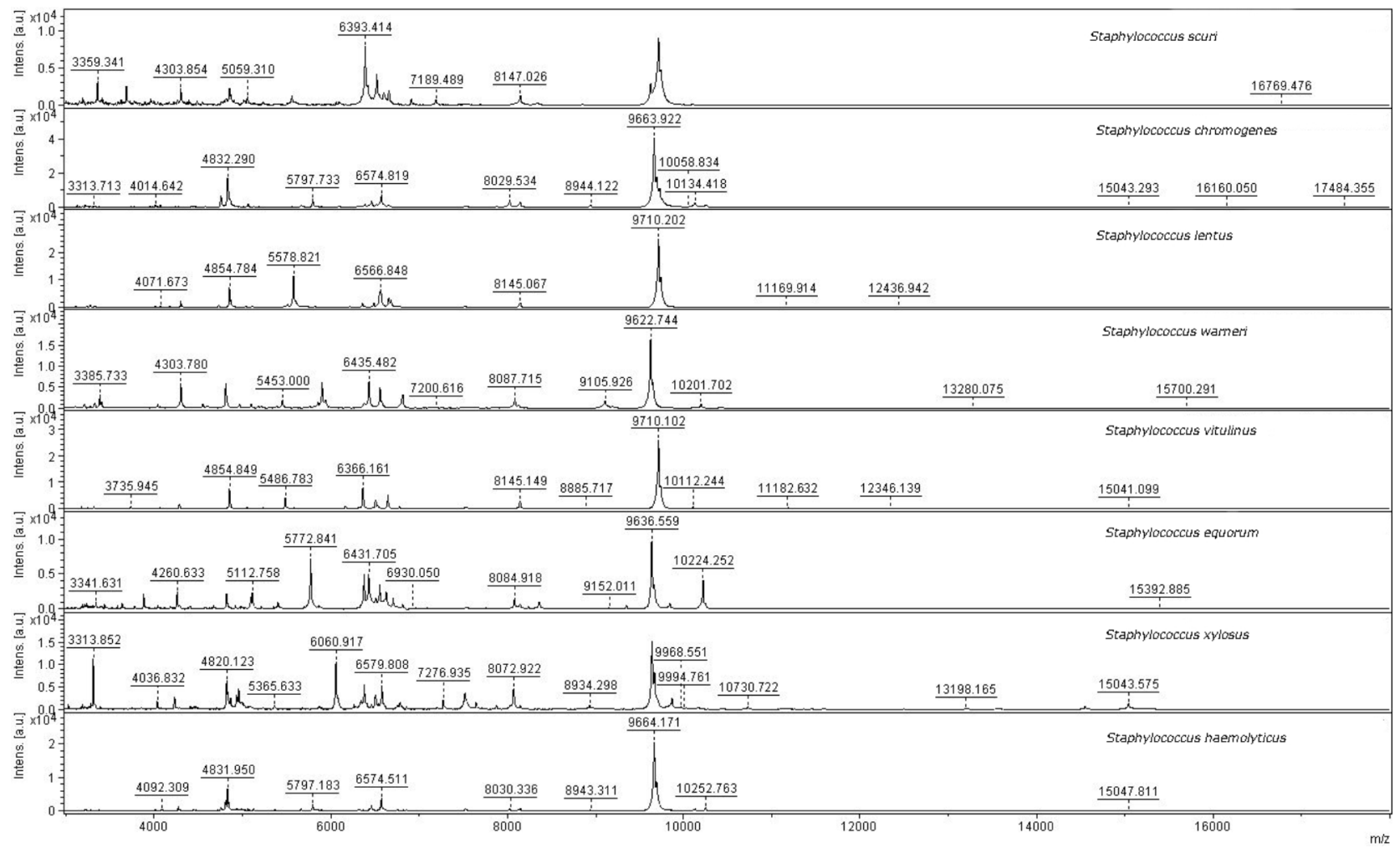

Fig. 1. Mass spectra of Staphylococcus spp isolated from milk of cows with subclinical mastitis.

a standard calibration mixture of an E. coli extract (Bruker Daltonik GmbH Bremen, Germany) containing RNase $\mathrm{A}$ and myoglobin proteins. The spectrum of the analyzed bacteria was obtained in a positive linear manner, within the mass range of 2 to $20 \mathrm{kDa}$. The analysis was repeated three times for each sample. The bacterial spectra were acquired using the flexControl 3.0 program, and then they were analyzed using a Bruker Biotyper 3.0. Samples with log (score) values above 2.0 were subjected to further analysis, which, according to the procedure, meant certain identification of the bacteria genus and highly probable identification of the species.

Standard strains of staphylococci were used as MALDI-TOF MS internal controls: $S$. sciuri ATCC 29062, S. lentus ATCC 29070, S. xylosus ATCC 29971, S. warneri ATCC 27836, S. chromogenes ATCC 43764, S. equorum ATCC 43958, and S. haemolyticus ATCC 29970.

\section{Results}

The coagulase-negative staphylococci determined with the API Staph tests were assigned to the following species: $S$. sciuri (21 strains $=64 \%)$, S. xylosus $(5$ strains $=15 \%), S$. chromogenes $(2$ strains $=6 \%)$, $S$. equorum (2 strains $=6 \%)$, S. lentus (1 strain
$=3 \%)$, S. warneri $(1$ strain $=3 \%)$ and S. haemolyticus $(1$ strain $=3 \%)$.

Based on the spectra analyses, the MALDI-TOF MS tests of 33 bacterial samples allowed identification of the microorganisms in 27 cases. Subclinical mastitis was caused by: $S$. sciuri (13 strains $=39 \%), S$. vitulinus (5 strains $=15 \%), S$. xylosus (3 strains $=9 \%), S$. chromogenes $(2$ strains $=6 \%)$, S. lentus $(1$ strain $=3 \%)$, S. warneri (1 strain $=3 \%)$, S. equorum $(1$ strain $=3 \%)$, and $S$. haemolyticus $(1$ strain $=3 \%)$. For each of the isolated microorganisms, the $\log$ (score) value of the analyzed spectra was above 2.0, whereas the log (score) values of the spectra obtained from the strains used as an internal control were in the range 2.7 (S. xylosus) to 2.9 (S. sciuri). The results of the bacterial identification process using MALDI-TOF MS are shown in Fig. 1.

Subsequent computer-assisted analysis of the obtained spectra resulted in a dendrogram showing the degree of similarity between the protein profiles of the isolated coagulase-negative staphylococci strains and the standard strains of staphylococci used as internal controls in the MALDI-TOF MS tests (Fig. 2). Its examination led to the identification of two monophyletic groups of coagulase-negative staphylococci. The first one consisted of the following species: S. xylosus and $S$. equorum, while the other included 


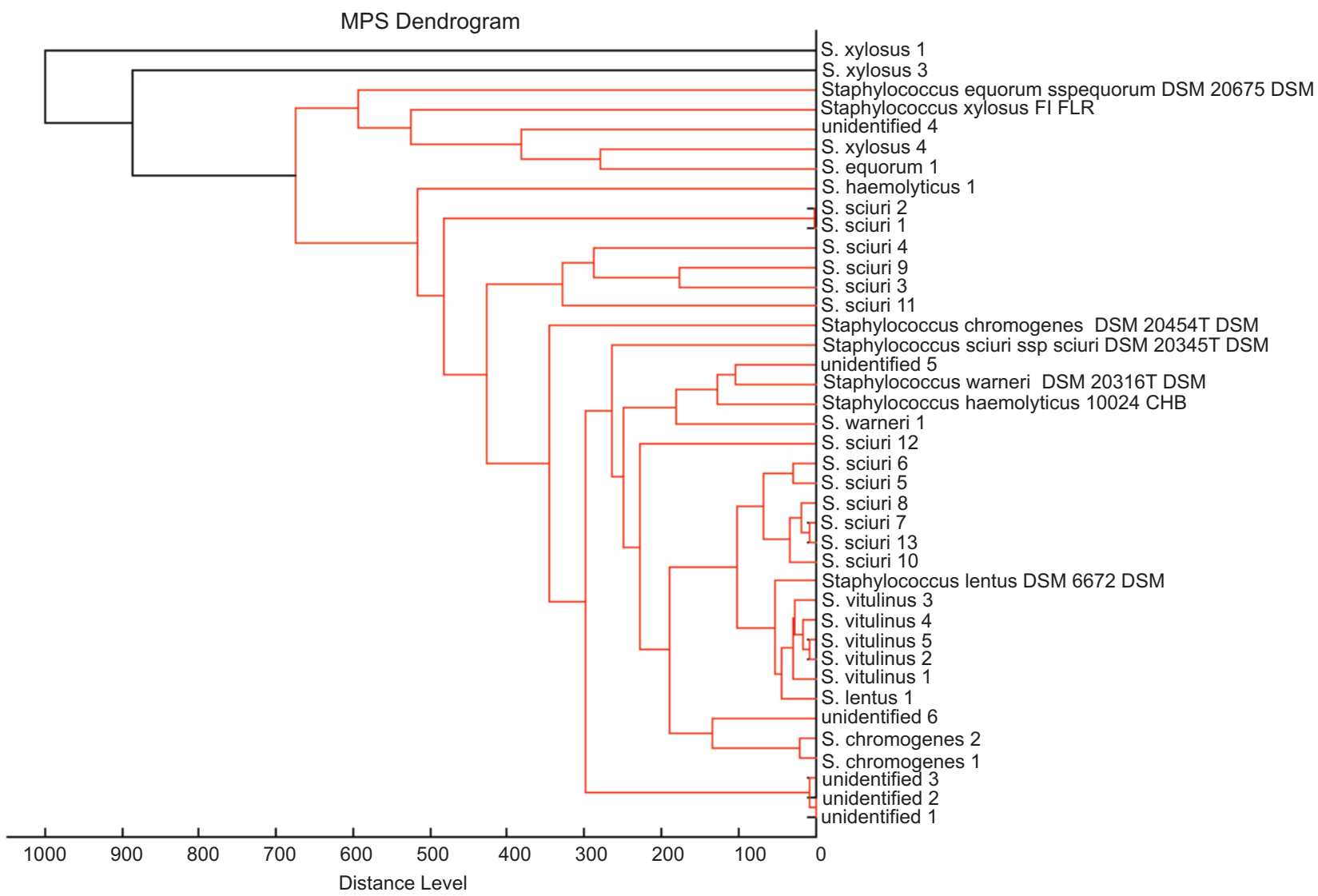

Fig. 2. The degree of similarity between protein profiles of the isolated Staphylococcus strains and standard strains of staphylococci used as internal controls in MALDI-TOF MS.

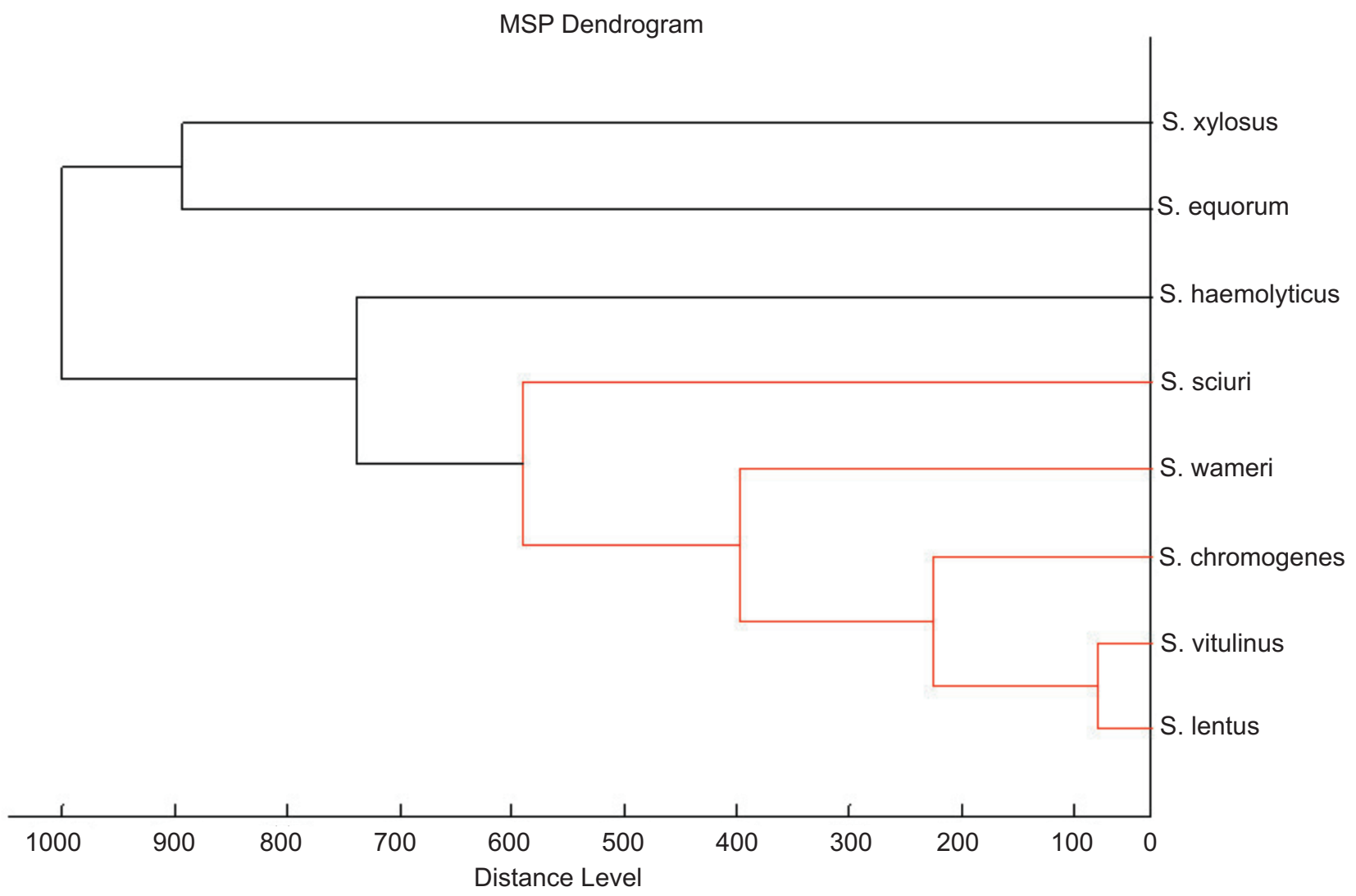

Fig. 3. Simplified version of the dendrogram presenting profiles of coagulase-negative staphylococci isolated from milk of cows with subclinical mastitis. 
S. haemolyticus, S. sciuri, S. warneri, S. chromogenes, S. vitulinus, and S. lentus. (Fig. 3).

Strains of bacteria not identified by MALDI-TOF MS were classified by means of routine bacteriological testing, and comprised: $S$. sciuri (3 strains), S. xylosus (2 strains), and S. equorum (1 strain).

\section{Discussion}

Despite being a very sensitive and reliable diagnostic technique, mass spectrometry does not represent a perfect test method. This is confirmed both by the present results and by literature data (Perreten et al. 2013). In six cases, the authors were unable to identify species of CNS causing subclinical mastistis using MALDI-TOF MS (log value less than 2.0). Similar results were obtained by Perreten et al. (2013), who were unable to determine the primary etiological factor of mastitis in $18 \%$ of disease cases using the PCR/ESI-MS technique. Other studies (Tomazi et al. 2014) demonstrated that the sensitivity of MALDI-TOF MS for the identification of CNS isolated from cow udders is $95.4 \%$ in comparison to PCR-RFLP. These observations show that MALDI-TOF MS, due to its lower sensitivity as compared to biochemical and molecular tests, can be used to identify staphylococci isolated both from clinical and subclinical cases of mastitis exclusively in order to supplement the above-mentioned techniques.

The score of the Biotyper system is an important factor in the identification of an isolate. A higher score indicates a higher confidence level and greater accuracy of identification. The manufacturer suggested a score $\geq 2.300$ was needed for secure species identification, and 2.000-2.299 for secure genus but probable species identification. However, in practice, lower scores have been suggested as acceptable for species level identification (Richter et al. 2012, Szabados et al. 2012). For example, one study with ethanol/formic acid extraction suggested using scores of $\geq 2.0$ for secure species identification of $S$. aureus, S. capitis, S. epidermidis, S. hominis, S. lugdunensis, S. saprophyticus, and $S$. simulans, and a score of 1.7 as sufficient for identification of S. caprae, S. sciuri, and $S$. cohnii (Richter et al. 2012). This suggests that the species- specific score cutoff values may require additional optimization or more extensive representation in the database (Zhu et al. 2015).

Many factors may contribute to the Biotyper score, such as cell wall rigidity, growth phase, and culture conditions, including selective media that may affect the protein expression. The concentration of cells observed has also been shown to influence the MALDI-TOF MS identification (Clark et al. 2013, Veloo et al. 2014). Applying approximately $1 \times 10^{7}$ bacterial cells from the $70 \%$ formic acid extraction onto the target plate resulted in better spectral resolution. The use of larger cell masses gave rise to spectral background noise resulting in no score or species ID. Too many cells may overwhelm the extraction of formic acid solution and hence the cells may not be properly disrupted (Zhu et al. 2015). In practice, it is not possible to estimate how many bacterial cells are presented in extracts, so this may be a cause of misidentification at the species level.

The present results correspond to those of other authors, and suggest that coagulase-negative staphylococci are the main cause of mastitis in cows, while $S$. xylosus, $S$. chromogenes, $S$. haemolyticus and $S$. sciuri are the species most commonly isolated from the milk of diseased animals (Bochniarz et al. 2013, Frey 2013). Staphylococcus equorum, S. warneri and $S$. lentus are less frequently present in such material according to the literature data (Anaya-López et al. 2006, Bochniarz et al. 2012). The available literature fails to offer data concerning subclinical infections in the udders of cows caused by $S$. vitilinus, even though in the present study these microorganisms were identified by MALDI-TOF MS in 15\% of the disease cases. These discrepancies may be explained by the fact that the discussed bacteria belong, together with $S$. lentus, to the $S$. sciuri group. Although biochemical tests like API Staph and ID32 STAPH make it possible to properly identify $S$. sciuri and $S$. lentus, they are unable to correctly determine $S$. vitulinus. Subspecies identification of these bacteria is only possible based on molecular (PCR) or proteomic (MALDI-TOF MS) tests (Stepanovic et al. 2005). Whenever the microbiological analysis of milk only involves culturing and identifying the isolated bacteria strains based on their biochemical properties, $S$. vitulinus can be misidentified as $S$. sciuri. Such a situation was observed in the present study. All strains of bacteria identified by MALDI-TOF MS as $S$. vitulinus were classified by API Staph as $S$. sciuri. These observations additionally confirm the usefulness of mass spectrometry in microbiological diagnostics.

The present study has shown that MALDI-TOF MS can be used not only to identify bacteria quickly, but it can also be used to test the degree of similarity between various coagulase-negative staphylococci species. Disclosing the presence of the two monophyletic groups of staphylococci demonstrates that this technique allows tracking of changes in the cell protein profiles of the microorganisms used in phylogenetic tests, and in many cases permits the 
simple detection of bacterial strains without the need to amplify and sequence their genetic material. This is important for both the taxonomy and epidemiology of human and animal infectious diseases, including infectious diseases of the bovine mammary gland.

\section{References}

Anaya-López JL, Contreras-Guzmán OE, Cárabez-Trejo A, Baizabal-Aguirre VM, López-Meza JE, Valdez-Alarcón JJ, Ochoa-Zarzosa A (2006) Invasive potential of bacterial isolates associated with subclinical bovine mastitis. Res Vet Sci 81: 358-361.

Anhalt JP, Fenselau C (1975) Identification of bacteria using mass spectrometry. Anal Chem 47: 219-225.

Biswas S, Rolain JM (2012) Use of MALDI-TOF mass spectrometry for identification of bacteria that are difficult to culture. J Microbiol Methods 92: 14-24.

Bizzini A, Greub G (2010) Matrix-assisted laser desorption ionization time-of-flight mass spectrometry, a revolution in clinical microbial identification. Clin Microbiol Infect 16: 1614-1619.

Bochniarz M, Wawron W (2012) Haemolytic and proteolytic activity of coagulase-negative staphylococci isolated from mastitis cows. Pol J Vet Sci 15: 61-65.

Bochniarz M, Wawron W, Szczubiał M (2013) Coagulase-negative staphylococci (CNS) as an aetiological factor of mastitis in cows. Pol J Vet Sci 16: 487-492.

Bright JJ, Claydon MA, Soufian M, Gordon DB (2002) Rapid typing of bacteria using matrix-assisted laser desorption ionization time-of-flight mass spectrometry and pattern recognition software. J Microbiol Methods 48: 127-138.

Bruker (2015) Extraction Method, MALDI Biotyper protocol guide, 3: 10-11.

Carbonnelle E, Mesquita C, Bille E, Day N, Dauphin B, Beretti JL, Ferroni A, Gutmann L, Nassif X (2010) MALDI-TOF mass spectrometry tools for bacterial identification in clinical microbiology laboratory. Clin Biochem 44: 104-109.

Clark AE, Kaleta EJ, Arora A, Wolk DM (2013) Matrix-assisted laser desorption ionization-time of flight mass spectrometry: a fundamental shift in the routine practice of clinical microbiology. Clin Microbiol Rev 26: 547-603.

Fox A (2006) Mass spectrometry for species or strain identification after culture or without culture: past, present, and future. J Clin Microbiol 44: 2677-2680.

Frey Y, Rodriguez JP, Thomann A, Schwendener S, Perreten V (2013) Genetic characterization of antimicrobial resistance in coagulase-negative staphylococci from bovine mastitis milk. J Dairy Sci 96: 2247-2257.

Malinowski E, Lassa H, Kłossowska A, Smulski S, Markiewicz H, Kaczmarowski M (2006) Etiological agents of dairy cow mastitis in western part of Poland. Pol J Vet Sci 9: 191-194.

Moon JS, Lee AR, Kang HM, Lee ES, Kim MN, Paik YH, Park YH, Joo YS, Koo HC (2007) Phenotypic and genetic antibiogram of methicillin-resistant staphylococci iso- lated from bovine mastitis in Korea. J Dairy Sci 90: 1176-1185.

Moser A, Stephan R, Ziegler D, Johler S (2013) Species distribution and resistance profiles of coagulase-negative staphylococci isolated from bovine mastitis in Switzerland. Schweiz Arch Tierheilkd 155: 333-338.

Perreten V, Endimiani A, Thomann A, Wipf JR, Rossano A Bodmer M, Raemy A, Sannes-Lowery KA, Ecker DJ, Sampath R, Bonomo RA, Washington C (2013) Evaluation of PCR electrospray-ionization mass spectrometry for rapid molecular diagnosis of bovine mastitis. J Dairy Sci 96: 3611-3620.

Pyörälä S, Taponen S (2009) Coagulase-negative staphylococci - emerging mastitis pathogens. Vet Microbiol 134: 3-8.

Richter C, Hollstein S, Woloszyn J, Kaase M, Gatermann SG, Szabados F (2012) Evaluation of species-specific score cut-off values for various Staphylococcus species using a MALDI Biotyper-based identification. J Med Microbiol 61: 1409-1416.

Seng P, Rolain JM, Fournier PE, La Scola B, Drancourt M, Raoult D (2010) MALDI-TOF-mass spectrometry applications in clinical microbiology. Future Microbiol 5: $1733-1754$.

Stepanovic S, Dakic I, Morrison D, Hauschild T, Jezek P, Petrhs P, Martel A, Vukovic D, Shittu A, Devriese LA (2005) Identification and characterization of clinical isolates of members of the Staphylococcus sciuri group. J Clin Microbiol 43: 956-958.

Szabados F, Tix H, Anders A, Kaase M, Gatermann SG, Geis G (2012) Evaluation of species-specific score cutoff values of routinely isolated clinically relevant bacteria using a direct smear preparation for matrix-assisted laser desorption/ionization time-of-flight mass spectrometry-based bacterial identification. Eur J Clin Microbiol Infect Dis 31: 1109-1119.

Taponen S, Koort J, Bjorkroth J, Saloniemi H, Pyorala $\mathrm{S}$ (2007) Bovine intramammary infections caused by coagulase-negative staphylococci may persist throughout lactation according to amplified fragment length polymorphism - based analysis. J Dairy Sci 90: 3301-3307.

Tomazi T, Gonçalves JL, Barreiro JR, de Campos Braga PA, Prada e Silva LF, Eberlin MN, dos Santos MV (2014) Identification of coagulase-negative staphylococci from bovine intramammary infection by matrix-assisted laser desorption ionization-time of flight mass spectrometry. J Clin Microbiol 52: 1658-1663.

Veloo AC, Elgersma PE, Friedrich AW, Nagy E, van Winkelhoff AJ (2014) The influence of incubation time, sample preparation and exposure to oxygen on the quality of the MALDI-TOF MS spectrum of anaerobic bacteria. Clin Microbiol Infect 20: O1091-O1097.

Waller KP, Aspan A, Nyman A, Persson Y, Andersson UG (2011) CNS species and antimicrobial resistance in clinical and subclinical bovine mastitis. Vet Microbiol 152: $112-116$.

Zhu W, Sieradzki K, Albrecht V, McAllister S, Lin W, Stuchlik O, Limbago B, Pohl J, Kamile Rasheed J (2015) Evaluation of the Biotyper MALDI-TOF MS system for identification of Staphylococcus species. J Microbiol Methods 117: 14-17. 